\title{
Ordered spectral statistics in 1D disordered supersymmetric quantum mechanics and Sinai diffusion with dilute absorbers
}

\author{
Christophe Texier \\ Univ. Paris Sud ; CNRS ; LPTMS, UMR 8626 \& LPS, UMR 8502 ; Orsay F-91405, \\ France \\ E-mail: christophe.texier@u-psud.fr
}

\begin{abstract}
Some results on the ordered statistics of eigenvalues for one-dimensional random Schrödinger Hamiltonians are reviewed. In the case of supersymmetric quantum mechanics with disorder, the existence of low energy delocalized states induces eigenvalue correlations and makes the ordered statistics problem nontrivial. The resulting distributions are used to analyze the problem of classical diffusion in a random force field (Sinai problem) in the presence of weakly concentrated absorbers. It is shown that the slowly decaying averaged return probability of the Sinai problem, $\overline{P(x, t \mid x, 0)} \sim \ln ^{-2} t$, is converted into a power law decay, $\overline{P(x, t \mid x, 0)} \sim t^{-\sqrt{2 \rho / g}}$, where $g$ is the strength of the random force field and $\rho$ the density of absorbers.

PACS numbers: 72.15.Rn ; 02.50.-r ; 05.40.-a
\end{abstract}




\section{Introduction}

An ordered (or extreme value) statistics problem can be defined as follows : given a set of $\mathcal{N}$ ranked random variables $x_{1}<x_{2}<\cdots<x_{n}<\cdots<x_{\mathcal{N}}$, one asks for the probability density $W_{n, \mathcal{N}}(x)$ for finding $x_{n}$ at $x$. When the random variables are independent and identically distributed (i.i.d) according to a given distribution $p(x)$, the distributions $W_{n, \mathcal{N}}(x)$ may be easily related to $p(x)$. The interesting point emerges when considering the $\mathcal{N} \rightarrow \infty$ limit : in this case, up to a rescalling $x_{n}=a_{n, \mathcal{N}}+b_{n, \mathcal{N}} y_{n}$, that depends on the original distribution $p(x)$, the distribution of the rescaled variable $y_{n}$ converges to an universal law $w_{n}(y)$. Three universality classes exist, corresponding to the nature of the tail of $p(x)$, as demonstrated in the pioneering works of Fréchet [7] and Gumbel (see the textbook [10]). When $p(x)$ has a tail of exponential type, one obtains the famous generalized Gumbel laws $w_{n}(y)=\frac{n^{n}}{(n-1) !} \exp n\left(y-e^{y}\right)$. The study of extreme events is relevant in many areas like meteorology, finance, computer science, statistical physics, etc, and in general the random variables of interest may not fulfill the hypothesis of statistical independence (see for example Ref. [18] or [1]). In this case the problem is much more difficult to analyze and no general results are known such as for i.i.d random variables. This situation occurs when considering the set of eigenvalues of a random operator, since eigenvalues are a priori correlated. For example, the distribution of the smallest eigenvalue of large random matrices has been obtained in a famous work by Tracy and Widom [27, 28] for the usual Gaussian ensembles ; other ensembles have been recently considered in Refs. [13, 14, 29, 21]. In the present paper, we will consider the case where random variables are eigenvalues of a one-dimensional (1D) Schrödinger operator with a random potential.

The paper is organised as follows : in section 2 we define the problem and review some results. In particular we will focus ourselves on the case of disordered supersymmetric quantum mechanics for which we obtain a set of non trivial distributions $w_{n}(y)$. Applications of these results are discussed in sections 3 and 4 : in the context of 1D disordered quantum mechanics and in the context of classical diffusion in a random force field with absorption.

\section{Ordered spectral statistics}

Let us consider the Schrödinger operator $H_{\text {scalar }}=-\frac{\mathrm{d}^{2}}{\mathrm{~d} x^{2}}+V(x)$ acting on functions defined on the interval $[0, L]$, satisfying Dirichlet boundary conditions $\varphi(0)=\varphi(L)=0$. We denote by $E_{1}<E_{2}<\cdots<E_{n}<\cdots$ the (infinite) set or eigenvalues. We will be interested in situations where the potential $V(x)$ is random. The section is devoted to the analysis of the probability density for finding the $n$-th eigenvalue $E_{n}$ at level $E$, denoted $W_{n}(E ; L)=\overline{\delta\left(E-E_{n}\right)}$, where $\cdots$ denotes averaging with respect with the disordered potential $V(x)$. The knowledge of this set of distributions provides some spectral informations much more precise than the averaged density of states (DoS) $\overline{\varrho(E ; L)}=\sum_{n=1}^{\infty} W_{n}(E ; L)$, measuring the probability to find any eigenvalue at level $E$. 
The determination of the distributions $W_{n}(E ; L)$ has been achieved for different types of random potentials, what we review in the rest of the section. We will consider the limit $L \rightarrow \infty$ (in practice $L$ must be larger than a certain scale charcateristic of the disorder) analogous to the $\mathcal{N} \rightarrow \infty$ limit of the introduction.

\subsection{Fully localized spectrum}

The distributions $W_{n}(E ; L)$ were determined when $V(x)$ is a Gaussian white noise : the distribution of the ground state energy $(n=1)$ was obtained in Ref. [19], a result generalized to all eigenvalues in Ref. [23]. The case of a potential describing the superposition of repulsive scatterers at random positions, $V(x)=\sum_{i} \alpha_{i} \delta\left(x-x_{i}\right)$, was considered in Ref. [9] ; $x_{i}$ are uncorrelated and uniformly distributed on $[0, L]$ with a mean density $\rho$; the positive weights $\alpha_{i}$ can be chosen random or not. These two models of random potential present different spectral densities and localization properties. They share however two facts [16] : ( $i)$ a suppression of the low energy DoS (Lifshits tails), compared to the free DoS, and ( $i$ i) a decreasing localization length as $E$ decreases (low energy states get more localized). This explains the origin of the result obtained in Refs. [9, 23] : after an appropriate model dependent rescaling of energies, $y_{n}=\left(E_{n}-\overline{E_{n}}\right) / \sqrt{\operatorname{var}\left(E_{n}\right)}$, the distributions $W_{n}(E ; L)$ coincide, in the limit $L \rightarrow \infty$, with the generalized Gumbel laws $w_{n}(y)$ aforementionned for uncorrelated variables. The absence of energy level correlations is explained by the strong localization of low energy states trapped in deep potential wells [20].

\subsection{Spectrum unfolding}

An interesting observation is related to the structure obtained in Ref. [23] for localized eigenstates in the $L \rightarrow \infty$ limit : $W_{n}(E ; L) \simeq L N^{\prime}(E) \frac{1}{(n-1) !}[L N(E)]^{n-1} \exp -L N(E)$, where $N(E)=\lim _{L \rightarrow \infty} \frac{1}{L} \int_{-\infty}^{E} \mathrm{~d} E^{\prime} \overline{\varrho\left(E^{\prime} ; L\right)}$ is the integrated DoS per unit length (IDoSpul) for $L=\infty$. The change of variable $\xi_{n}=L N\left(E_{n}\right)$ corresponds to unfolding the spectrum : it relates a set of random variables $\left\{E_{n}\right\}$ distributed with a non-uniform density $\overline{\varrho(E ; L)} \simeq L N^{\prime}(E)$, to random variables $\left\{\xi_{n}\right\}$ distributed with a uniform density equal to unity. The fact that these latter variables are distributed according to a Poisson

law $\varpi_{n}(\xi)=\frac{1}{(n-1) !} \xi^{n-1} e^{-\xi}$ demonstrates the absence of energy level correlations [20], since $\varpi_{n}(\xi)$ is also the probability that $n-1$ eigenvalues lie in the interval $[0, \xi]$.

\subsection{Supersymmetric case}

The results reviewed in the previous subsection apply to the generic situation where all eigenstates are localised. However this does not exhaust all possible scenarii, as there exist one-dimensional disordered models exhibiting delocalized states. Such an example is provided by the supersymmetric quantum Hamiltonian

$$
H_{\text {susy }}=-\frac{\mathrm{d}^{2}}{\mathrm{~d} x^{2}}+\phi(x)^{2}+\phi^{\prime}(x)=Q^{\dagger} Q
$$


where $Q=-\frac{\mathrm{d}}{\mathrm{d} x}+\phi(x)$. This Hamiltonian appears in many interesting contexts (see Refs. [5, 4, 25] for reviews). When $\phi(x)$ has short range correlations and $\overline{\phi(x)}=0$, we obtain low energy properties opposite to the one mentioned previously for $H_{\text {scalar }}$ : $(i)$ the disorder increases the low energy DoS (Dyson singularity) and (ii) the states get delocalized near the spectrum boundary at $E=0$ (this was demonstrated in Ref. [2] when $\phi(x)$ is a Gaussian white noise). The delocalization of the first eigenstates are responsible for energy level correlations and the distributions $W_{n}(E ; L)$ are no longer expected to coincide with the generalized Gumbel laws. We now explain the principle of the method, introduced in Ref. [23], allowing for the determination of these distributions, when $\phi(x)$ is a Gaussian white noise such that $\overline{\phi(x)}=0$ and $\overline{\phi(x) \phi\left(x^{\prime}\right)}=g \delta\left(x-x^{\prime}\right)$.

The starting point consists in converting the Sturm-Liouville (spectral) problem, $H_{\text {susy }} \varphi(x)=E \varphi(x)$ with boundary conditions $\varphi(0)=\varphi(L)=0$, into a Cauchy problem, $H_{\text {susy }} \psi(x ; E)=E \psi(x ; E)$ with boundary conditions $\psi(0 ; E)=0$ and $\psi^{\prime}(0 ; E)=1$. The former admits solutions only for energy in a discrete set $E \in\left\{E_{n}\right\}$, while the latter has solutions $\forall E \in \mathbb{R}$. We introduce the notation $\ell_{1}, \ell_{2}$, etc, for the lengths between the consecutive nodes of the wave function (Fig. 1), and $P(\ell)$ their common distribution, that will be obtained by studying the statistical properties of $\psi(x ; E)$, or rather of some related Riccati variable. Then the probability for the eigenenergy $E_{n}$ to be at level $E$ coincides with the probability for the $n$-th zero to coincide with the boundary $\sum_{m=1}^{n} \ell_{m}=L$, i.e. $W_{n}(E ; L) \propto(P * \cdots * P)(L)$, as illustrated on Fig. 11. This is the essence of the node counting method, also denoted phase formalism in the context of disordered systems [16] (or Dyson-Schmidt method for discrete models [17]).

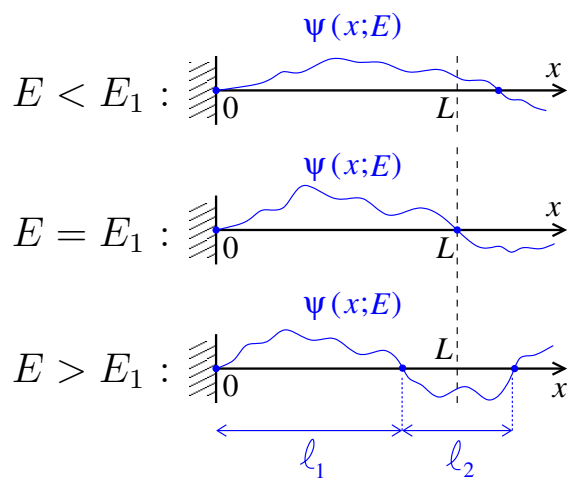

Figure 1. Solution of the Cauchy problem for different energies, below, equal and above the ground state energy.

The building brick is now the distribution $P(\ell)$ and we explain how it can be calculated. Introducing the Riccati variable $z=\psi^{\prime} / \psi-\phi$, we map the Schrödinger equation onto a Langevin like equation

$$
\frac{\mathrm{d}}{\mathrm{d} x} z(x)=-E-z(x)^{2}-2 z(x) \phi(x) .
$$

In this language the distance $\ell_{n}$ separates two consecutive divergencies of the Riccati variable, e.g. $z(0)=+\infty$ and $z\left(\ell_{1}\right)=-\infty$. The study of $P(\ell)$ is therefore mapped onto 
a first passage problem for a stochastic Markovian process, a problem solved by wellknown techniques. We introduce the auxiliary "time" $\mathcal{L}_{z}$ needed by the process $z(x)$ in order to reach $-\infty$ for the first time, given that it has started at $z$. The characteristic function $h(z ; \alpha)=\overline{e^{-\alpha \mathcal{L}_{z}}}$ obeys a backward Fokker-Planck equation

$$
\mathcal{B}_{z} h(z ; \alpha)=\alpha h(z ; \alpha)
$$

for boundary conditions $h(-\infty ; \alpha)=1$ and $h^{\prime}(+\infty ; \alpha)=0$, where $\mathcal{B}_{z}=2 g z \frac{\mathrm{d}}{\mathrm{d} z} z \frac{\mathrm{d}}{\mathrm{d} z}-(E+$ $\left.z^{2}\right) \frac{\mathrm{d}}{\mathrm{d} z}$ is the generator of the diffusion. Finally the distribution $P(\ell)$ can be obtained by an inverse Laplace transform

$$
h(+\infty ; \alpha)=\int_{0}^{\infty} \mathrm{d} \ell P(\ell) e^{-\alpha \ell} .
$$

An approximation scheme was proposed in Ref. 23] in order to solve Eq. (3) in the low energy regime $E \ll g^{2}$. This supposes that the distributions $W_{n}(E ; L)$ are mostly concentrated on such energy scales, what is expected to occur when $L \rightarrow \infty$. As a result, it was shown that

$$
h(+\infty ; \alpha) \simeq \frac{1}{\cosh ^{2} \sqrt{\alpha / N_{\text {susy }}(E)}}
$$

where $1 / \bar{\ell}=N_{\text {susy }}(E) \simeq 2 g / \ln ^{2}\left(g^{2} / E\right)$ is the IDoSpul of the supersymmetric Hamiltonian (1) in bulk $(L=\infty)$. After spectrum unfolding $\xi_{n}=L N_{\text {susy }}\left(E_{n}\right)$, the distributions of the eigenvalues $W_{n}(E ; L)=L N_{\text {susy }}^{\prime}(E) \varpi_{n}\left(L N_{\text {susy }}(E)\right)$ are given by

$$
\varpi_{n}(\xi)=\int_{\mathscr{B}} \frac{\mathrm{d} q}{2 \mathrm{i} \pi} \frac{e^{q \xi}}{\cosh ^{2 n} \sqrt{q}}
$$

where $\mathscr{B}$ is a Bromwich contour. The fact that these distributions deviate from the Poisson distribution (i.e. $W_{n}(E ; L)$ differ from the generalized Gumbel laws) signals spectral correlations induced by delocalization. These distributions were explicitely calculated in Ref. [25] by a generating function method :

$$
\varpi_{n}(\xi)=\frac{2^{2 n}}{\sqrt{\pi} \xi^{3 / 2}} \sum_{m=n}^{\infty}(-1)^{n+m} m\left(\begin{array}{c}
m+n-1 \\
m-n
\end{array}\right) e^{-m^{2} / \xi} .
$$

The large $\xi$ behaviour is $\varpi_{n}(\xi) \simeq \frac{\pi^{2 n}}{(2 n-1) !} \xi^{2 n-1} \exp -\frac{\pi^{2}}{4} \xi$. The first distributions are represented on Fig. 2.

The sum of the distributions characterizes the averaged DoS

$$
\overline{\varrho(E ; L)}=\sum_{n=1}^{\infty} W_{n}(E ; L)
$$

for a finite interval. It coincides to the bulk density of states $\overline{\varrho(E ; L)} \simeq L N_{\text {susy }}^{\prime}(E)$ when eigenstates are localized on scale smaller than $L$ and do not feel the boundaries. A log-normal depletion, $\overline{\varrho(E ; L)} \sim \frac{1}{E \sqrt{g L}} \exp -\frac{1}{2 g L} \ln ^{2}\left(g^{2} / E\right)$, is however obtained at low energy $E \ll g^{2} \exp -\sqrt{2 g L}$, illustrating the sensitivity of low energy delocalized states to the boundaries. The DoS is represented on Fig. 2 (dashed line) after spectrum unfolding. Conversely the boundary sensitivity of the averaged DoS $\overline{\varrho(E ; L)}$ may be 
used as a localization criterium, what gives the energy dependence of the localization length $\xi_{E} \sim \frac{1}{g} \ln ^{2}\left(g^{2} / E\right)$.

Details on the results reviewed in this section can be found in Refs. [23, 25].
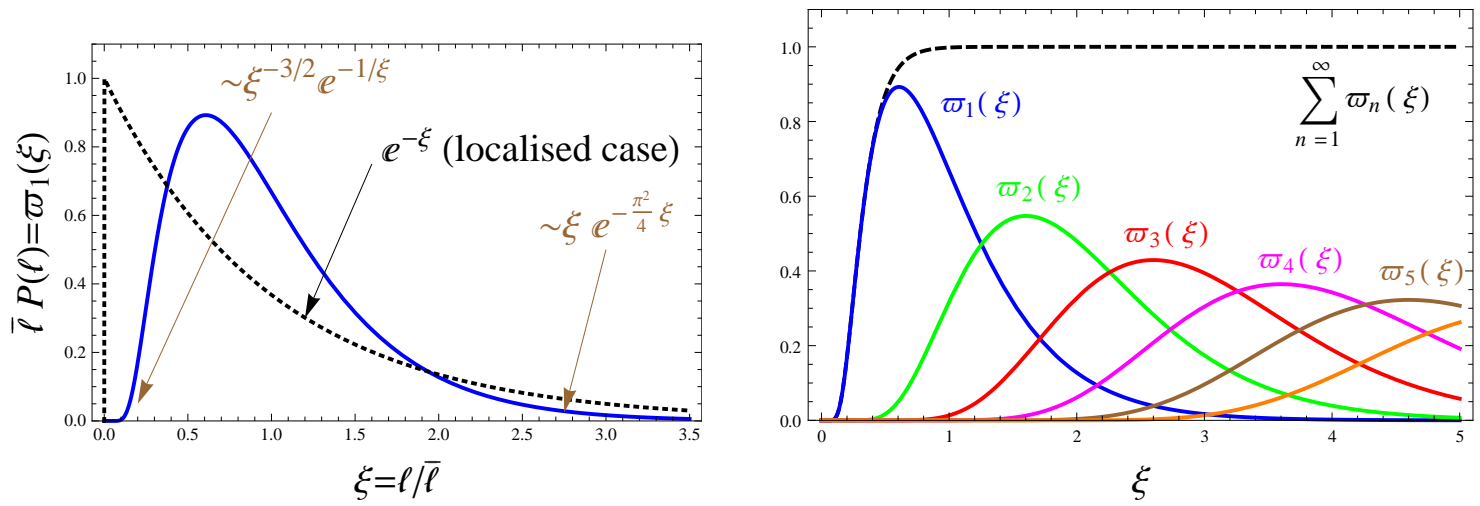

Figure 2. Left: The distribution $\varpi_{1}(\xi)$ may be interpreted as the distribution of the rescaled distance $\ell$ between consecutive nodes of the wave function $P(\ell)=\varpi_{1}(\ell / \bar{\ell}) / \bar{\ell}$; the low energy wave function of the SUSY Hamiltonian therefore exhibits repulsion between nodes. For reference, we have also plotted (dotted black) the exponential distribution (absence of repulsion between nodes) characterizing localised eigenstates, the case discussed in $\S 2.183$ 2.2. Right : Distributions $\varpi_{n}(\xi)$ of the five first rescaled eigenvalues $\xi_{n}=L N_{\text {susy }}\left(E_{n}\right)$. The sum (dashed line) corresponds to the averaged DoS in a finite interval, after spectrum unfolding with respect to bulk DoS; depletion near $\xi=0$ is due to the delocalization.

\section{Supersymmetry broken by disorder}

As we have mentioned, the random Hamiltonians $H_{\text {scalar }}$ and $H_{\text {susy }}$ present opposite spectral and localization properties. This observation has led to question the interplay between the two types of disorder and consider the mixed case

$$
H=-\frac{\mathrm{d}^{2}}{\mathrm{~d} x^{2}}+\phi(x)^{2}+\phi^{\prime}(x)+V(x) .
$$

The case where $\phi$ and $V$ are two Gaussian white noises was analyzed in Ref. [11], however it turns out that the case where the potential $V$ describes a random superposition of repulsive scatterers at random positions, $V(x)=\sum_{i} \alpha_{i} \delta\left(x-x_{i}\right)$, can be related to an interesting problem in the context of classical diffusion (discussed in next section). In this latter case, the spectral density of the Hamiltonian (9) may be obtained from the distributions $W_{n}(E ; L)$ obtained in the previous section by using a Lifshits like argument [24]. Let us denote by $N(E)$ the IDoSpul of the Hamiltonian (9). In the limit of large weights $\alpha_{i} \rightarrow \infty$ (in practice $\alpha_{i} \gg \rho \& g$ ) the impurities decouple the intervals free of impurity and impose Dirichlet boundary conditions at their locations. Thus the spectrum of $(9)$ is given the addition of spectra of $H_{\text {susy }}$ on all intervals and we obtain the DoSpul $N^{\prime}(E)$ for the mixed disorders by averaging the DoS (8) of the 
supersymmetric Hamiltonian for a finite length

$$
N^{\prime}(E) \simeq \rho\langle\overline{\varrho(E ; L)}\rangle_{L},
$$

where $\langle\cdots\rangle_{L}$ denotes averaging with respect to the length of interval, namely with an exponential law $\rho e^{-\rho L}$ characterizing uncorrelated positions $x_{i}$. Using (68) we get

$$
N(E) \simeq \frac{\rho}{\sinh ^{2} \sqrt{\rho / N_{\text {susy }}(E)}} .
$$

Above the threshold energy $E_{c}=g^{2} \exp -\sqrt{2 g / \rho}$, the IDoS coincides with the one of the supersymmetric Hamiltonian. Below the threshold, the Dyson singularity is transformed into a power-law singularity

$$
N(E) \underset{\rho \rightarrow 0}{\simeq} 4 \rho\left(\frac{E}{g^{2}}\right)^{\sqrt{2 \rho / g}} .
$$

This power law behaviour has been very well confirmed by numerical calculations [24]. Moreover the numerics has shown that the exponent $\sqrt{2 \rho / g}$ seems more general than the range of application of the Lifshits argument suggests, what we will comment on in the last section.

\section{Sinai diffusion with weakly concentrated absorbers}

The study of classical diffusion in a random environment has attracted a lot of attention in various contexts ranging from mathematics, statistical physics (glassy dynamics or polymer physics) and even finance. It may be described within the framework of Fokker-Planck equation $\partial_{t} P(x ; t)=\partial_{x}\left(\partial_{x}-2 \phi(x)\right) P(x ; t)$, where $\phi(x)$ is chosen to be a Gaussian white noise with $\overline{\phi(x)}=\mu g$ and $\overline{\phi(x) \phi\left(x^{\prime}\right)}-\overline{\phi(x)} \overline{\phi\left(x^{\prime}\right)}=$ $g \delta\left(x-x^{\prime}\right)$. The model exhibits a rich dynamics as a function of the drift $\mu[2$. In the absence of drift, for $\mu=0$, the diffusion is controlled by overcoming of potential barriers and is extremely slow $x(t) \sim \frac{1}{g} \ln ^{2}\left(g^{2} t\right)$ (Sinai diffusion [22]).

The question I would like to discuss now is : how does the introduction of absorbing sites affect the Sinai dynamics ? Let us go back for a moment to the free diffusion : in this case the return probability decays in time as a power law $P(x, t \mid x, 0)=1 / \sqrt{4 \pi t}$. The dynamics is slowed down by the introduction of a random force field, what is reflected

in the return probability by an extremely slow decay $\overline{P(x, t \mid x, 0)} \sim g \ln ^{-2}\left(g^{2} t\right)$, related to the behaviour aforementioned. On the other hand, when a weak concentration $\rho$ of efficient absorbers with large absorbing rates $\alpha \gg \rho$ is introduced, the free power law decay is transformed into an exponential decay $\overline{P(x, t \mid x, 0)} \sim \exp -3\left(\frac{\pi^{2}}{4} \rho^{2} t\right)^{1 / 3}$ (Lifshits tail). The question is : what is the behaviour of the return probability when both random force field and absorbers are present ? Is the return probability increased or decreased?

The answer to this question can be obtained by a simple mapping to the model analyzed in the previous section. The effect of absorbers on the Sinai diffusion may be accounted for by adding to the Fokker-Planck equation a term :

$$
\partial_{t} P(x ; t)=\partial_{x}\left(\partial_{x}-2 \phi(x)\right) P(x ; t)-V(x) P(x ; t)
$$


where $V(x)=\sum_{i} \alpha_{i} \delta\left(x-x_{i}\right)$ describes absorbers located at a set of positions $\left\{x_{i}\right\}$. The positive coefficient $\alpha_{i}$ measures the efficiency of the absorber located at $x_{i}$. The Fokker-Planck equation (13) may be mapped onto the Schrödinger equation $-\partial_{t} \psi(x ; t)=H \psi(x ; t)$ for the Hamiltonian (9) thanks to the transformation $P(x ; t)=$ $\psi(x ; t) \exp \int^{x} \mathrm{~d} x^{\prime} \phi\left(x^{\prime}\right)$. It follows that the return probability can be related to the spectral density (DoSpul) $\rho(E)=N^{\prime}(E)$ of Hamiltonian (9) by

$$
\overline{P(x, t \mid x, 0)}=\int_{0}^{\infty} \mathrm{d} E \rho(E) e^{-E t} .
$$

We immediately deduce the large time behaviour of the return probability from (12) :

$$
\overline{P(x, t \mid x, 0)} \sim t^{-\sqrt{2 \rho / g}} \text { for } t \gg t_{c},
$$

where the scale $t_{c}=1 / E_{c}=\frac{1}{g^{2}} \exp \sqrt{2 g / \rho}$ is the time needed by the particle moving in the random force field to reach the closest absorber $x\left(t_{c}\right) \sim 1 / \rho$ where $x(t) \sim \frac{1}{g} \ln ^{2}\left(g^{2} t\right)$. The power-law can be mostly explained by analyzing the lowest absorption rate $E_{1}[\phi(x), L]$ in a finite interval : the fact that its distribution presents a log-normal behaviour $W_{1}(E ; L) \sim \frac{1}{E} \exp -\frac{1}{2 g L} \ln ^{2}\left(g^{2} / E\right)$ for $E \rightarrow 0$ explains the exponent. The behaviour (15) was first obtained in Ref. [24] and later confirmed in Ref. [15] by the real space renormalization group method. The return probability decays slower than in the free case for a sufficiently weak concentration of absorbers $\rho<g / 8$, whereas it decays faster for $\rho>g / 8$. Note however that the origin of the decay in the present model is mostly due to absorption, as demonstrated in Ref. [15] by analyzing the survival probability which was shown to present the same power law decay up to a logarithmic correction $\overline{S_{x_{0}}(t)}=\int \mathrm{d} x \overline{P\left(x, t \mid x_{0}, 0\right)} \sim(\ln t) t^{-\sqrt{2 \rho / g}}$.

\section{Concluding remarks}

In this brief review, I have analyzed the ordered statistics (extreme value statistics) for eigenvalues of a random supersymmetric Hamiltonian. The fact that this quantum Hamiltonian possesses delocalized low energy eigenstates makes the problem nontrivial and escape from the universal generalized Gumbel laws obtained for generic 1D random Hamiltonians with a full spectrum of localized states.

These results have found some application in order to analyze the effect of dilute absorbers on the classical diffusion in a random environment (Sinai diffusion). We have obtained that the return probability presents a power law decay, mostly due to absorption. This conclusion relies on the analysis of the spectral density for a mixed random Hamiltonian with a "supersymmetric part" $\phi^{2}+\phi^{\prime}$, where $\phi(x)$ is a Gaussian white noise, and a "scalar part" $V(x)=\sum_{i} \alpha_{i} \delta\left(x-x_{i}\right)$. Using a Lifshits like argument, it was shown that the DoS of this Hamiltonian presents a power law singularity at low energy $\rho(E)=N^{\prime}(E) \sim E^{-1+\sqrt{2 \rho / g}}$. The result is interesting by itself since only few power law spectral singularities are known in the context of 1D Anderson localization : (i) the Halperin singularity, for randomly dropped attractive impurities of fixed weights $\alpha_{i}=-v$ [12, 16] : $\rho(E) \sim\left|E+\frac{1}{4} v^{2}\right|^{-1+2 \rho / v}$ for $E \sim-\frac{1}{4} v^{2}$. (ii) The power law 
behaviour for the supersymmetric Hamiltonian $H_{\text {susy }}$ for a finite $\overline{\phi(x)}=\mu g \neq 0$ for which $\rho(E) \sim E^{-1+\mu}[2]$.

Surprisingly, numerical simulations of the model (9) revealed that the exponent appearing in the IDoSpul $(12)$ is much more robust than one would have expected on the basis of the heuristic Lifshits argument. Examining this observation has led us to a solvable version of the model by considering random weights $\alpha_{i}$. Let us consider the Hamiltonian (9) for a finite drift $\overline{\phi(x)}=\mu g \neq 0$ and with exponentially distributed positive weights $p\left(\alpha_{i}\right)=\frac{1}{v} \exp -\frac{\alpha_{i}}{v}$. This model becomes solvable for a particular value of the parameter $v=\overline{\alpha_{i}}$ : if $v=2 g$ the effect of the "scalar term" $V(x)$ can be absorbed in a redefinition of the parameter $\mu[26]$

$$
\mu \longrightarrow \nu=\sqrt{\mu^{2}+\frac{2 \rho}{g}}
$$

The IDoS of $(9)$ can be obtained by performing this substitution in the IDoS of $H_{\text {susy }}$ (that can be found in Ref. [2]), therefore :

$$
N(E)=\frac{2 g}{\pi^{2}} \frac{1}{J_{\nu}(\sqrt{E} / g)^{2}+N_{\nu}(\sqrt{E} / g)^{2}},
$$

where $J_{\nu}(x)$ and $N_{\nu}(x)$ are Bessel functions [8]. Using their asymptotic behaviours we obtain the low energy IDoS $N(E) \simeq \frac{2 g}{\Gamma(\nu)^{2}}\left(\frac{E}{4 g^{2}}\right)^{\nu}$ for $E \ll g^{2}$. Therefore we have recovered

the power law with exponent $\sqrt{2 \rho / g}$ (setting $\mu=0$ ) within a solvable model, confirming the robustness of this exponent valid for arbitrary value of the ratio $\rho / g$. It is worth emphasizing that the solvable model applies to a regime with weights $\alpha_{i} \sim g$ while the analysis of the previous sections rather relied on the hypothesis that $\alpha_{i} \gg g$, $\rho$. This result also generalizes the analysis to finite drift $\mu \neq 0$ (note that the same exponent $\sqrt{\mu^{2}+2 \rho / g}$ was obtained by Le Doussal in Ref. [15] by another method, in a different regime $\left.\alpha_{i} \rightarrow \infty\right)$. Details of this analysis will be published in a forthcoming article [26].

\section{Acknowledgements}

The material presented here results from collaborations with Alain Comtet, Christian Hagendorf and Yves Tourigny to whom I address my deep thanks. I am grateful to Satya Majumdar for helpful remarks and suggestions. Nordita Institute at Stokholm is acknowledged for his hospitality during the program "Fundations and Applications of non-equilibrium statistical mechanics" (october 2011), financially supported by the European Science Fundation.

\section{Appendix A. Diffusion constant of a single $d$-dimensional Brownian curve}

In Ref. [25], the distribution of the maximal height of a Brownian excursion was shown to be related to the distributions $(6 / 7)$, or more precisely to their sum $\sum_{n=1}^{\infty} \varpi_{n}(\xi)$.

In this appendix, we point out that the distributions

$$
\pi_{n}(\xi)=\int_{\mathscr{B}} \frac{\mathrm{d} q}{2 \mathrm{i} \pi} \frac{e^{q \xi}}{\cosh ^{n} \sqrt{q}},
$$


generalizing $\varpi_{n}(\xi)=\pi_{2 n}(\xi)$ and considered in Ref. [25], are relevant in order to characterize a certain property of the $d$-dimensional Brownian motion.

Let us consider a $d$-dimensional Brownian curve $\vec{r}(\tau)$ with $\tau \in[0, t]$ starting from the origin $\vec{r}(0)=\overrightarrow{0}$. This curve is weighted according to the Wiener measure

$$
\mathcal{D} \vec{r}(\tau) e^{-\frac{1}{4 D} \int_{0}^{t} \mathrm{~d} \tau\left(\frac{\mathrm{d} \vec{r}(\tau)}{\mathrm{d} \tau}\right)^{2}}
$$

where $D$ is the diffusion constant, usually defined by $D \stackrel{\text { def }}{=} \lim _{t \rightarrow \infty} \frac{1}{2 t d} \mathbb{E}\left(\vec{r}(t)^{2}\right)$, where $\mathbb{E}(\cdots)$ denotes the expectation with respect to the Wiener measure A.2. We now ask the question : is it possible to obtain an estimation of the diffusion constant from a given realization of the Brownian path by a time average ? For this purpose we introduce the "trajectory-dependent diffusion constant" $\mathscr{D}_{t} \stackrel{\text { def }}{=} \frac{1}{t d} \int_{0}^{t} \frac{\mathrm{d} \tau}{t} \vec{r}(\tau)^{2}$, or preferably the dimensionless Brownian functional

$$
\chi[\vec{r}(\tau)] \stackrel{\text { def }}{=} \frac{\mathscr{D}_{t}}{D}=\frac{1}{D t d} \int_{0}^{t} \frac{\mathrm{d} \tau}{t} \vec{r}(\tau)^{2} .
$$

Note that this problem was also considered in Ref. [3], starting from a different definition of the trajectory-dependent diffusion constant $\int_{0}^{t} \frac{\mathrm{d} \tau}{t} \frac{1}{2 d \tau} \vec{r}(\tau)^{2}$.

The characteristic function of the functional $\chi[\vec{r}]$ may be written thanks to a path integral

$$
\Gamma(p) \stackrel{\text { def }}{=} \mathbb{E}\left(e^{-p \chi[\vec{r}(\tau)]}\right)=\int \mathrm{d} \vec{R} \int_{\vec{r}(0)=\overrightarrow{0}}^{\vec{r}(t)=\vec{R}} \overrightarrow{\vec{r}}(\tau) e^{-\int_{0}^{t} \mathrm{~d} \tau\left[\frac{1}{4 D} \dot{\vec{r}}(\tau)^{2}+\frac{p}{D t^{2} d} \vec{r}(\tau)^{2}\right]}
$$

We recognize the integral of the imaginary time propagator $\left\langle\vec{R}\left|e^{-t H}\right| \overrightarrow{0}\right\rangle$ for a quantum harmonic oscillator with mass $m \rightarrow 1 /(2 D)$ and pulsation $\omega \rightarrow \frac{2}{t} \sqrt{\frac{p}{d}}$. Using its wellknown expression [6] we straightforwardly obtain

$$
\Gamma(p)=\frac{1}{\cosh ^{\frac{d}{2}}(2 \sqrt{p / d})} .
$$

We check that $\mathbb{E}(\chi[\vec{r}])=1$ as it should. The variance is given by $\operatorname{Var}(\chi[\vec{r}])=\frac{4}{3 d}$ and vanishes in the limit of large dimension, as expected from the central limit theorem. In the case of even dimensions, we recognize the Laplace transform of the distribution A.1). Therefore we deduce that the distribution of the functional $\chi[\vec{r}]$ is

$$
P_{d}(\chi)=\frac{d}{4} \pi_{\frac{d}{2}}\left(\frac{d}{4} \chi\right) \quad \text { for dimension } d \text { even . }
$$

Expressions of the distributions $\pi_{n}(\xi)$ can be found in Ref. [25].

\section{References}

[1] G. Biroli, J.-P. Bouchaud and M. Potters, Extreme value problems in random matrix theory and other disordered systems, J. Stat. Mech. P07019 (2007).

[2] J.-P. Bouchaud, A. Comtet, A. Georges and P. Le Doussal, Classical diffusion of a particle in a one-dimensional random force field, Ann. Phys. (N.Y.) 201, 285-341 (1990).

[3] D. Boyer, D. S. Dean, C. Mejía-Monasterio and G. Oshanin, Optimal estimates of the diffusion coefficient of a single Brownian trajectory, Phys. Rev. E 85, 031136 (2012). 
[4] A. Comtet, J. Desbois and C. Texier, Functionals of the Brownian motion, localization and metric graphs, J. Phys. A: Math. Gen. 38, R341-R383 (2005).

[5] A. Comtet and C. Texier, One-dimensional disordered supersymmetric quantum mechanics: a brief survey, in Supersymmetry and Integrable Models, edited by H. Aratyn, T. D. Imbo, W.-Y. Keung and U. Sukhatme, Lecture Notes in Physics, Vol. 502, pp. 313-328. Springer (1998), Proceedings of a workshop held at Chicago, IL, USA, 12-14 June 1997 (also available as cond-mat/97 07 313).

[6] R. P. Feynman and A. R. Hibbs, Quantum mechanics and path integrals, McGraw-Hill (1965).

[7] M. Fréchet, Sur la loi de probabilité de l'écart maximum, Ann. Soc. Polonaise Math. Cracovie 6, 93 (1927).

[8] I. S. Gradshteyn and I. M. Ryzhik, Table of integrals, series and products, Academic Press, fifth edition (1994).

[9] L. N. Grenkova, S. A. Molčanov and Ju. N. Sudarev, On the basic states of one-dimensional disordered structures, Commun. Math. Phys. 90(1), 101-123 (1983).

[10] E. J. Gumbel, Statistics of Extremes, Columbia University Press, New York (1958).

[11] C. Hagendorf and C. Texier, Breaking supersymmetry in a one-dimensional random Hamiltonian, J. Phys. A: Math. Theor. 41, 405302 (2008).

[12] B. I. Halperin, Properties of a particle in a one-dimensional random potential, Adv. Chem. Phys. 13, 123-177 (1967).

[13] K. Johansson, Shape fluctuations and random matrices, Commun. Math. Phys. 209, 437-476 (2000).

[14] I. M. Johnstone, On the distribution of the largest eigenvalue in principal component analysis, Ann. Statist. 29(2), 295-327 (2001).

[15] P. Le Doussal, Sinai model in presence of dilute absorbers, J. Stat. Mech. P07032 (2009).

[16] I. M. Lifshits, S. A. Gredeskul and L. A. Pastur, Introduction to the theory of disordered systems, John Wiley \& Sons (1988).

[17] J.-M. Luck, Systèmes désordonnés unidimensionnels, CEA, collection Aléa Saclay, Saclay (1992).

[18] S. N. Majumdar and P. L. Krapivsky, Extreme Value Statistics and Traveling Fronts: Various Applications, Physica A 318, 161-170 (2003).

[19] H. P. McKean, A Limit Law for the Ground State of Hill's Equation, J. Stat. Phys. 74(5/6), 1227 (1994).

[20] S. A. Molčanov, The local structure of the spectrum of the one-dimensional Schrödinger operator, Commun. Math. Phys. 78(3), 429-446 (1981).

[21] C. Nadal, S. N. Majumdar and M. Vergassola, Statistical distribution of quantum entanglement for a random bipartite state, J. Stat. Phys. 142(2), 403-438 (2011).

[22] Ya. G. Sinai, The limit behavior of random walks in a one-dimensional random environment, Theory of Prob. and Appl. 27(2), 247 (1982).

[23] C. Texier, Individual energy level distributions for one-dimensional diagonal and off-diagonal disorder, J. Phys. A: Math. Gen. 33, 6095-6128 (2000).

[24] C. Texier and C. Hagendorf, One-dimensional classical diffusion in a random force field with weakly concentrated absorbers, Europhys. Lett. 86, 37011 (2009).

[25] C. Texier and C. Hagendorf, Effect of boundaries on the spectrum of a one-dimensional random mass Dirac Hamiltonian, J. Phys. A: Math. Theor. 43, 025002 (2010).

[26] C. Texier and Y. Tourigny, in preparation (2012).

[27] C. A. Tracy and H. Widom, Level-spacing distribution and the Airy kernel, Commun. Math. Phys. 159, 151-174 (1994).

[28] C. A. Tracy and H. Widom, On orthogonal and symplectic matrix ensembles, Commun. Math. Phys. 177, 727-754 (1996).

[29] P. Vivo, S. N. Majumdar and O. Bohigas, Large Deviations of the Maximum Eigenvalue in Wishart Random Matrices, J. Phys. A: Math. Theor. 40(16), 4317-4337 (2007). 